\title{
10. Gesundheitspflege-Kongress 26. und 27. Oktober 2012, Hamburg
}

Online publiziert: 14. Mai 2012

(C) Springer-Verlag 2012

\section{HeilberufeSCIENCE-Symposium}

Springer Medizin, führender Anbieter von Fachinformationen im Gesundheitswesen im deutschsprachigen Raum, gibt seit 2008 das wissenschaftliche peer-reviewed Online-Journal HeilberufeSCIENCE heraus.

Im Rahmen des 10. Gesundheitspflege-Kongresses 2012 in Hamburg findet zum dritten Mal das HeilberufeSCIENCE-Symposium statt. In Vorträgen und einer Postersession sollen wieder aktuelle wissenschaftliche Beiträge aus den Disziplinen Pflegemanagement, Pflegepädagogik, Pflegewissenschaften, Public Health, Gerontologie und Altenhilfe präsentiert werden.

Eingereicht werden können Abstracts zu wissenschaftlichen Studien, Abschlussarbeiten oder Ergebnissen aus Praxisprojekten - orientiert an den Kongressthemen oder zu einem frei gewählten Thema.

Schwerpunktthemen des 10. GesundheitspflegeKongresses u. a.

- Hygiene

- Onkologie

- Berufspolitik

- Kommunikation

- Akademisierung

- Führungsinstrumente

- Übertragung ärztlicher Tätigkeiten.

\section{Abstract}

- Umfang: 250 Worte (ohne Titel und Autoren)

- Format: WORD, um redaktionelle Bearbeitung zu ermöglichen

- Gliederung: Titel, AutorInnen/Institution(en), Einleitung/Fragestellung, Methodik/Stichprobe, Ergebnisse, Diskussion/Schlussfolgerungen - ohne Quellenangaben und ohne Tabellen/Abbildungen

- Schriftleitung und Redaktion von HeilberufeSCIENCE wählen die Beiträge für die Posterpräsentation bzw. Vorträge im 3. HeilberufeSCIENCE-Symposium aus

- Die Abstracts der ausgewählten Beiträge werden zitierfähig in einem HeilberufeSCIENCE-Supplement publiziert.

Einsendung bis 31. Juli 2012 per E-Mail an

Prof. Dr. med. habil. Jörg Klewer FRSPH

Schriftleitung HeilberufeSCIENCE

E-Mail: heilberufescience@springer.com

Weitere Kongressinformationen

Springer Medizin • Urban \& Vogel GmbH •

Kongressorganisation

E-Mail: andrea.tauchert@springer.com

Internet: www.heilberufe-kongresse.de 